\title{
Development
}

\section{Sex and Pubertal Differences in the Maturational Trajectories of Sleep Spindles in the Transition from Childhood to Adolescence: A Population-Based Study}

\author{
Anna Ricci, ${ }^{1}$ Fan He, ${ }^{2}$ Susan L. Calhoun, ${ }^{1}$ Jidong Fang, ${ }^{1}$ Alexandros N. Vgontzas, ${ }^{1}$ Duanping Liao, ${ }^{2}$ \\ Edward O. Bixler, ${ }^{1}$ Magdy Younes, ${ }^{3}$ and Julio Fernandez-Mendoza ${ }^{1}$
}

https://doi.org/10.1523/ENEURO.0257-21.2021

${ }^{1}$ Sleep Research and Treatment Center, Department of Psychiatry and Behavioral Health, Penn State College of Medicine, Hershey, PA17033, ²Department of Public Health Sciences, Penn State College of Medicine, Hershey, PA17033, and ${ }^{3}$ Sleep Disorders Centre, University of Manitoba, Winnipeg, ManitobaR3M 0A7, Canada

\begin{abstract}
Sleep spindles, bursts of electroencephalogram (EEG) activity in the $\sigma$-frequency $(11-16 \mathrm{~Hz})$ range, may be biomarkers of cortical development. Studies capturing the transition to adolescence are needed to delineate age-related, sex-related, and pubertal-related changes in sleep spindles at the population-level. We analyzed the sleep EEG of 572 subjects $6-21$ years ( $48 \%$ female) and 332 subjects $5-12$ years (46\% female) followed-up at $12-22$ years. From 6 to 21 years, spindle density $(p$ quadratic $=0.019)$ and fast $(12-16 \mathrm{~Hz})$ spindle percent $(p$ quadratic $=0.016)$ showed inverted U-shaped trajectories, with plateaus after 15 and 19 years, respectively. Spindle frequency increased $(p$ linear $<0.001$ ), while spindle power decreased $(p$ linear $<0.001)$ from 6 to 21 years. The trajectories of spindle density, frequency, and fast spindle percent diverged between females and males, in whom density plateaued by 14 years, fast spindle percent by 16 years, and frequency by 18 years, while fast spindle percent and spindle frequency continued to increase until 21 years in females. Males experienced a longitudinal increase in spindle density $31 \%$ greater than females by $12-14$ years $(p=0.006)$. Females experienced an increase in spindle frequency and fast spindle percent $2 \%$ and $41 \%$ greater, respectively, than males by $18-22$ years (both $p=0.004$ ), while males experienced a $14 \%$ greater decline in spindle power by $18-22$ years $(p=0.018)$. Less mature adolescents $(86 \%$ male) experienced a longitudinal increase in spindle density $36 \%$ greater than mature adolescents by $12-14$ years $(p=0.002)$. Overall, males experience greater maturational changes in spindle density in the transition to adolescence, driven by later pubertal development, and sex differences become prominent in early adulthood when females have greater spindle power, frequency, and fast spindle percent.
\end{abstract}

Key words: adolescence; brain maturation; childhood; developmental biology; puberty; sleep spindles

\section{Significance Statement}

Age-related changes in sleep spindles reflect maturation of the thalamocortical network. We provide evidence that spindle metrics follow distinct developmental trajectories from each other and previously described sleep oscillations shown to index brain maturation in the transition to adolescence. Importantly, we report novel data regarding the association between spindle activity and pubertal development. Specifically, we found that less mature adolescents ( $86 \%$ male) experienced a greater increase in spindle density in the transition to ages 12-14 years, while more mature adolescents ( $75 \%$ female) experienced a greater decline in $\sigma$ /spindle power by the same age. These data suggest that females, who enter pubertal development earlier than males, may also begin brain maturational processes in the thalamocortical network at an earlier stage than males. 


\section{Introduction}

Sleep spindles occur as synchronized bursts of sinusoidal waves in the $\sigma$-frequency range $(11-16 \mathrm{~Hz})$ and are electroencephalogram (EEG) hallmarks of stage 2 (N2) of non-rapid eye movement (NREM) sleep (Rechtschaffen and Kales, 1968; Iber et al., 2007). Spindles are generated in the thalamus and synchronized in the cortex and thus, reflect activity of the thalamocortical network (Steriade et al., 1985; Steriade,1999). A predominant role of spindles is protecting the sleeping brain from external sensory stimuli, serving as biomarkers of sleep integrity (Dang-Vu et al., 2010, 2015; Saletin et al., 2013). Moreover, age-related changes in spindle activity may signal maturation of the thalamocortical network (D'Atri et al., 2018; Guadagni et al., 2021). It has also been suggested that spindles support cognitive function by preventing sleep fragmentation, allowing for better off-line information processing (e.g., memory consolidation), which is critical during early development (Fogel and Smith, 2011; Gruber and Wise, 2016). Indeed, spindles have been associated with cognitive processes in children and adolescents (Geiger et al., 2011; Chatburn et al., 2013; Hoedlmoser et al., 2014); however, such relationship differs across the lifespan (Reynolds et al., 2018). As sleep spindles are purported EEG biomarkers of neurodevelopment that represent the strength/integrity of the thalamocortical network, it is essential to examine their maturational trajectories and potential sex differences across specific developmental stages. These data will inform and expand the knowledge of sleep spindles as they relate to cognition and neurodevelopment in youth.

The majority of previous studies on the maturational trajectories of sleep spindles have relied on $\sigma$ power as a surrogate marker of spindle activity (Gaudreau et al., 2001; Campbell et al., 2005; Tarokh and Carskadon, 2010; Tarokh et al., 2011; Baker et al., 2012, 2016; PrehnKristensen et al., 2013; Olbrich et al., 2017). Campbell and Feinberg (2016) found a longitudinal increase in $\sigma$ power from 6 to 12 years, followed by a decline from 12 to 16 years $(n=92)$. While these $\sigma$ power trajectories may

Received June 7, 2021; accepted June 10, 2021; First published June 21, 2021.

J.F. discloses ownership of Biosoft Studio (Hershey, PA), which developed and maintains sleep FFT software. M.Y. discloses having developed, and having a patent on, the spindle analysis technology, which has been licensed to Cerebra Health (Winnipeg, Manitoba, Canada), and he is a shareholder and receives royalties and consultation fees from Cerebra Health. All other authors declare no competing financial interests.

Author contributions: A.R. and J.F.-M. designed research; A.R., F.H., J.F., M.Y., and J.F.-M. performed research; F.H. and D.L. analyzed data; A.R., F.H., S.L.C., J.F., A.N.V., D.L., E.O.B., M.Y., and J.F.-M. wrote the paper.

This work was supported by the National Institute of Mental Health; National Heart, Lung, and Blood Institute; and the National Center for Advancing Translational Sciences of the National Institutes of Health under Grants R01MH118308, R01HL136587, R01HL97165, R01HL63772, and UL1TR000127.

Correspondence should be addressed to Julio Fernandez-Mendoza at ifmendoza@psu.edu.

https://doi.org/10.1523/ENEURO.0257-21.2021 Copyright (C) 2021 Ricci et al.

This is an open-access article distributed under the terms of the Creative Commons Attribution 4.0 International license, which permits unrestricted use, distribution and reproduction in any medium provided that the original work is properly attributed. generalize to spindles, studies examining age-related changes specifically for spindles suggest that different metrics undergo distinct developmental trajectories (Bòdizs et al., 2014; Nader and Smith, 2015; McClain et al., 2016). Hahn et al. (2019) observed a longitudinal increase in spindle density and frequency that appeared to be driven by fast $(13-15 \mathrm{~Hz})$ spindles in the transition from 8-11 to $14-18$ years $(n=34)$. Although Hahn et al. (2019) did not examine sex or pubertal differences, Goldstone et al. (2019) found that females had higher fast $(\sim 13 \mathrm{~Hz})$ spindle frequencies than males, and more mature adolescents had greater fast spindle density than less mature adolescents in their cross-sectional sample $(n=134,12-21$ years). A more recent study found higher spindle amplitude, frequency, and density in 31 girls compared with 30 boys (914 years), suggesting greater thalamocortical coherence in females than males (Markovic et al., 2020). Finally, an extensive analysis of spindle activity $(11-15 \mathrm{~Hz})$ in 11630 individuals (Purcell et al., 2017) found distinct life-course trajectories and sex differences in spindle metrics from ages 4-97, including 448 children (4-10 years) with obstructive sleep apnea followed-up after six months, a cross-sectional sample of 509 adolescents (16-19 years), and a cross-sectional family sample with 183 youth (7-23 years). Purcell et al. (2017) produced seminal data from a life-course perspective; however, studies are still needed to expand on their findings by examining cohorts with greater representation of early adolescents undergoing puberty and longer longitudinal followup periods capturing the developmental transition to adolescence.

The aim of the present study was to improve the knowledge of age, sex, and pubertal differences in sleep spindles and related $\sigma$ activity during this developmental period by examining sex differences in 572 subjects aged 6-21 years, and sex-related and pubertal-related differences in 332 subjects aged 5-12 years followed-up 613 years later at ages $12-22$ years. As different spindle characteristics may represent specific features of cortical development, we examined spindle density, frequency, and power, as well as the percent of fast spindles. Spindle density may reflect thalamocortical coherence/connectivity (Steriade et al., 1985), while spindle frequency polarization of thalamocortical neurons (Goldstone et al., 2019), spindle power white matter integrity around the thalamus (Fernandez and Lüthi, 2020), and fast spindles hippocampal development (Saletin et al., 2013). Thus, examining each of these metrics is essential to understand neurodevelopmental changes in sleep spindles. We compared the trajectories of spindle metrics to $\sigma$ power as it has been used as a surrogate measure of spindle activity. While we expect females to have greater spindle activity than males as indicated by previous studies (Purcell et al., 2017), we also hypothesize that age-related changes in spindle activity will be associated with pubertal development.

\section{Materials and Methods}

\section{Penn State child cohort (PSCC)}

The PSCC is a randomly-recruited sample of 700 children $(47.7 \%$ female, $23.7 \%$ racial/ethnic minority) from 
the general population who underwent a comprehensive in-lab study between ages 5 and 12 (median 9 years; Bixler et al., 2008; Calhoun et al., 2014). Out of the 700 children, 421 returned 6-13 years later (median 7.4 years) for a follow-up study when they were 12-23 years (median 16 years, $46.1 \%$ female, $21.9 \%$ racial/ethnic minority; Bixler et al., 2016; Fernandez-Mendoza et al., 2016, 2017). A total of 279 subjects (median 9 years, $47.8 \%$ female, $29.3 \%$ racial/ethnic minority) did not return for the follow-up study but were not significantly different from the 421 who were followed-up in their demographic characteristics at ages 5-12 (Bixler et al., 2016). All subjects or parents/legal guardians provided informed written consent for the study protocol, which was approved by Penn State's Institutional Review Board.

\section{Demographics and clinical measures}

During baseline and follow-up visits, the sleep study consisted of a clinical history, physical examination, selfreported questionnaires and a one-night, 9-h polysomnography (PSG) recording, all of which occurred in sound-controlled, light-controlled, and temperature-controlled rooms. Sex, race, ethnicity, and date of birth were reported during the clinical history at baseline. Height and weight were measured during the physical examination and body mass index (BMI) percentile was calculated (Kuczmarski et al., 2002). A 3-h standardized neurobehavioral assessment was performed at both time points (Achenbach, 1991; Wechsler, 1991, 1997, 1999, 2003; Achenbach and Rescorla, 2003). During the clinical history, parents at baseline and/or subjects at follow-up reported on the presence of a lifetime history of any psychiatric or behavioral disorder (Frye et al., 2018). Medication use was reported by parents at baseline and/or by subjects at follow-up during the clinical history and on an evening questionnaire (Frye et al., 2018). Psychotropic medication use was defined by a report of stimulants, antidepressants, anxiolytics, antipsychotics and/or hypnotics ( $n=58$ at baseline and $n=67$ at follow-up). At follow-up, Tanner stage was ascertained via a self-rating scale (Carskadon and Acebo, 1993) providing stages 1 (prepuberty), 2 (early puberty), 3 (mid puberty), 4 (late puberty), and 5 (adulthood).

\section{PSG}

Registered PSG technicians (RPSGTs) applied electrodes to the subject's scalp, face, and legs at 9 P.M. All sleep data were recorded for $9 \mathrm{~h}$ of time in bed from the time of "lights out" (9-11 P.M.) until the time of "lights on" (6-8 A.M.) to accommodate to the subject's habitual sleep schedule. All sleep data at baseline and follow-up were recorded using Grass PSG equipment (GrassTelefactor) and included EEG, electrooculography, electromyography, electrocardiography (ECG) and respiratory measures. All PSG recordings were visually scored by RPSGTs in 30-s epochs following standard criteria (Rechtschaffen and Kales, 1968; Iber et al., 2007; Bixler et al., 2008, 2016). Out of the 700 recordings at baseline (B), 48 were performed on paper PSG and six digital records were corrupted, thus, their EEG could not be analyzed. All
421 recordings at follow-up $(F)$ were performed on digital PSG and were analyzable.

There were unavoidable PSG system updates during this large, long-term study that collected baseline data across fouryears and follow-up data 6-13 years later across three years. Given these PSG updates there were differences in the number of EEG channels, adding other referencing methods, filter settings, and sampling rates, all of which were accounted for in the spectral analyses as well as in the statistical analyses by controlling for the PSG system (see below, Statistical analyses). Specifically, a total of 373 baseline files (B1) were recorded with a sampling rate of $100 \mathrm{~Hz}$ and filter settings at $0.1-100.0 \mathrm{~Hz}$. The remaining 273 baseline files (B2) were recorded with sampling rates at 100.0 and $200.0 \mathrm{~Hz}$ and filter settings at $0.01-30.0 \mathrm{~Hz}$. Importantly, there were no significant differences at baseline between subjects recorded with PSG system B1 versus B2 in terms of critical demographic factors, including distribution of male sex $(49.6 \%$ vs $52.3 \%, p=0.700)$ and age $(8.4 \pm 1.7$ vs $8.5 \pm 1.7, p=0.799)$. All of the follow-up PSG files $(F)$ were recorded with a sampling rate of $200 \mathrm{~Hz}$ and filter settings at $0.1-70.0 \mathrm{~Hz}$. All PSG data were automatically processed with spectral analysis software with the common EEG frequencies ranging between 0.3 and $30.0 \mathrm{~Hz}$, thus, activities below and above this range were removed for consistent data processing across all European data format (EDF) records from different PSG systems. Additionally, not all subjects had available central derivations with contralateral referencing and spectral analyses for those subjects were conducted using the central derivations with ipsilateral referencing used at the time of the PSG (only 122 in cross-sectional analyses and 167 in longitudinal analyses in the present study). Among the subset of records that had both contralateral and ipsilateral channels, we found excellent concordance in both the cross-sectional sample [C3M1 vs C3-M2, $n=105, \rho_{\mathrm{C}}=0.985,95 \%$ confidence interval $(95 \% \mathrm{Cl})=0.979,0.990 ; \mathrm{C} 4-\mathrm{M} 1$ vs $\mathrm{C} 4-\mathrm{M} 2$, $\left.n=109, \rho_{\mathrm{c}}=0.983,95 \% \mathrm{Cl}=0.975,0.988\right]$ and longitudinal sample (C3-M1 vs C3-M2, $n=188, \rho_{\mathrm{c}}=0.997$, $95 \% \mathrm{Cl}=0.996-0.998 ; \mathrm{C} 4-\mathrm{M} 1$ vs C4-M2, $n=190, \rho_{\mathrm{C}}=$ $0.996,95 \% \mathrm{Cl}=0.995,0.997$ ) assuring reliability of the data.

All 646 baseline and 421 follow-up $(N=1067)$ digital PSG records were converted into EDF files and analyzed in a blind manner using two independent systems: Michele Sleep Scoring (MSS; Cerebra Health, Sleep Disorders Centre, University of Manitoba, Winnipeg, Canada) and sleepFFT (Biosoft Studio, Pennsylvania State University, Hershey, PA). MSS analyzed central sleep spindles and sleepFFT central $\sigma$ power.

\section{Sleep spindles}

All sleep EEGs were analyzed with MSS through a data use agreement between J.F.M. (Pennsylvania State University) and M.Y. (University of Manitoba). De-identified EDF files were securely shared with M.Y. blind of any demographic, clinical or date of recording data to assure blindness and rigor of EEG data processing. MSS 
provided spindle metrics (density, frequency, power, and fast spindle percent) for N2 sleep in central derivations (C3 and C4), and were identified in the 10.0 to $16.0 \mathrm{~Hz}$ range. Details on this validated software can be found elsewhere (Guadagni et al., 2021; Goldschmied et al., 2020). In brief, the fast Fourier transform (FFT) was applied to 1-s epochs with the window advancing every 0.2 $s$ (five windows per second). The sum of power in the spindle frequency range (power S) was calculated in each $1-s$ epoch. The spindle frequency range was defined as 10.0-16.0 Hz. Power $S$ in each $1-$ s epoch was divided by the 30th percentile of power $S$ in all 1-s epochs within each 30-s epoch (power $S$ ratio). A spindle was identified when the power $S$ ratio was $>3$ for five consecutive epochs provided the ratio decreased to $<1.5$, or power $S$ decreased to $<20 \%$ of peak power S (whichever was higher) within $5 \mathrm{~s}$ (Goldschmied et al., 2020). Presumptive spindles were deleted if they occurred during arousals and if the power $S$ ratio within the presumptive spindle was less than the ratios of $\alpha$ and $\beta$ powers to their respective reference values (30th percentile in the 30-s epoch). Spindle density was calculated as the total number of spindles in N2 sleep and divided by the time in minutes of N2 sleep. Spindle frequency was measured as the frequency with the highest power in the 10.0 to $16.0 \mathrm{~Hz}$ range and expressed in Hertz. Spindle power was defined as the highest power $S$ within the spindle and expressed as microvolts squared $\left(\mu \mathrm{V}^{2}\right)$. Fast spindles were identified in the 12.0 to $16.0 \mathrm{~Hz}$ range and expressed as the percent of fast spindles out of all identified spindles. Averages of spindle density, frequency, power, and fast spindle percent were calculated in N2 for central derivations.

\section{$\sigma$ power}

All sleep EEGs were also analyzed with sleepFFT software (Fernandez-Mendoza et al., 2016, 2019; Ricci et al., 2021) in which the FFT was used to estimate absolute NREM (N2 and N3) $\sigma$ power. $\sigma$ power was defined as frequencies in the 11.33 to $14.84 \mathrm{~Hz}$ range, consistent with previous studies using the frequency range of $11-15 \mathrm{~Hz}$ (Tarokh et al., 2011; Campbell and Feinberg, 2016; Purcell et al., 2017). $\sigma$ power was analyzed in addition to spindle metrics to increase the rigor and reproducibility of the present study as it has been used in previous studies as a surrogate measure of spindle density and may follow a similar age-related trajectory. The same central EEG derivations (C3 and C4) were analyzed in all artifact-free NREM sleep epochs. All EDF files underwent thorough systematic procedures for rejecting EEG epochs with movement artifacts, correcting ECG interference intruding into EEG channels, sorting spectral data according to visually scored sleep stages, and calculation of EEG power during sleep/wake states using the FFT with correction for rejected epochs. We verified our automatic processing methods by visual inspection at epoch-by-epoch levels and compared automatic processing to visual inspection results in 1063 subjects (643 baseline and 420 follow-up) to assure the rigor and reproducibility of our data. Trained technicians examined the automatic processing results at an epoch-by-epoch level and had the ability to make corrections. The results of visual inspection by trained technicians were compared with automatic processing results in 1063 subjects, which resulted in high concordance (0.93-1.0), indicating that these methods highly agree with each other. We also determined the percent of epochs included in the analyses across all subjects, which was 85-90\% across all sleep stages and channels. Therefore, automatic processing provided valid representations of our data that agree with human visual inspection.

All-night spectral analysis was performed on visually scored 30-s epochs. All overnight PSG data were automatically processed in a single run with sleepFFT by a trained graduate assistant blind of any of the subject's characteristics. As mentioned above, the common EEG frequencies ranged from $0.3-30.0 \mathrm{~Hz}$ and activities below and above this range were removed for consistent data processing across all EDF records from different $P S G$ systems (B1, B2, and F). SleepFFT used eight orders of Butterworth band pass filter with a high pass filter set at $0.3 \mathrm{~Hz}$ and a low pass filter set at $30.0 \mathrm{~Hz}$. Each $30-\mathrm{s}$ epoch was applied with 22 overlapping Hann windows lasting $2.56 \mathrm{~s}$, with overlaps between windows by approximately half. The FFT was performed on each overlapping window to generate power density data with $0.39 \mathrm{~Hz}$ resolution. The resulting data were averaged across these 22 windows as the power spectral data for the epoch. Absolute NREM (N2 and N3) $\sigma$ power was computed by summing the power density data (including lower and upper limits of the frequency band), adjusting for rejected epochs, averaging for $\mathrm{C} 3$ and $\mathrm{C} 4$, and expressed as $\mu \mathrm{V}^{2}$.

\section{Statistical analyses}

In order to examine the age-related trajectories of spin$\mathrm{dle} / \sigma$ activity we derived a cross-sectional sample spanning from age 5 to 23 by aggregating independent subjects who contributed with data at ages 5-12 years ( $n=279)$ and at ages $12-23$ years $(n=421)$ with no subject represented twice. After excluding participants who were recorded on paper PSG $(n=27)$, whose EDF file was corrupted $(n=3)$, who had missing spectral data or were outliers $(n=6)$, or were taking psychotropic medications $(n=92), 572$ subjects were included in the cross-sectional analyses. Multivariable-adjusted linear regression models regressed subjects' age against spindle/ $\sigma$ activity. Age was treated as a continuous variable and truncated at 6 and 21 years because only two subjects were 5 years and eight were $\geq 22$ years. Given that previous studies (Nader and Smith, 2015; McClain et al., 2016; Purcell et al., 2017) found different, nonlinear age trajectories for specific spindle metrics, we tested nonlinear associations between age and spindle/ $\sigma$ activity by including quadratic and cubic terms in the models, along with the lower-order terms. The highest-ordered significant $(p<0.05)$ age term was used as the final model. The population-level means and their $95 \% \mathrm{Cl}$ of spindle/ $\sigma$ activity between ages 6 and 21 , estimated based on the final model, were plotted to represent the cross-sectional age-related trajectories. Covariates adjusted for in these models included sex, race/ethnicity, BMI, apnea/hypopnea index (AHI), psychiatric/learning disorder and PSG system (coded as B1 =0, 
$B 2=1, F=2$ and treated as a nominal factor). Sex-specific distributions were plotted for the highest significant ordered term. Furthermore, we estimated the age at which minimum and maximum predictive values in spindle/ $\sigma$ activity were reached. Piece-wise linear regression analyses were performed to obtain standardized regression coefficients $\left(\beta_{\mathrm{s}}\right)$ in the association between age and spindle/ $\sigma$ activity. Spindle/ $\sigma$ activities were divided by their own standard deviation to obtain the $\beta_{\mathrm{s}}$ and their standard error (SE). Sex differences in mean spindle/ $\sigma$ activity were also tested using analysis of covariance at developmentally appropriate piece-wise age segments [6-10 (childhood, $n=188$ ), 11-14 (early adolescence, $n=108$ ), 15-17 (mid-adolescence, $n=162$ ), and 18-21 (late adolescence/ early adulthood, $n=114)$ ].

In order to study the magnitude of longitudinal change in spindle $/ \sigma$ activity in the transition from childhood to adolescence, we focused on a sample of 332 subjects who had analyzable sleep EEG data at ages 5-12 years (baseline) and at ages 12-22 years (follow-up) and were not taking psychotropic medications. The within-subject change in spindle/ $\sigma$ activity between baseline and followup was the dependent variable in these longitudinal analyses and was calculated as a percent change with the formula: [(follow-up value - baseline value)/baseline value] $\times 100$. General linear models were used to calculate the age-related percent change in spindle/ $\sigma$ activity as a function of the following age groups: 12-14 years (early adolescence, $n=75$ ), $15-17$ years (mid-adolescence, $n=160$ ), and 18-22 years (late adolescence/early adulthood, $n=97$ ). Covariates adjusted for in these longitudinal models included sex, race/ethnicity, BMI, AHI, psychiatric/learning disorders, baseline PSG system (coded as $\mathrm{B} 1=0, \mathrm{~B} 2=1$ and treated as a binary factor), baseline spindle/ $\sigma$ activity, and length of follow-up (years elapsed between baseline and follow-up). By examining the longitudinal trajectory using this approach, we calculated the change in spindle $/ \sigma$ activity in the transitions from baseline mean age of 6.6 years to follow-up age 1214 years, from 8.7 to $15-17$ years and from 10.3 to $18-22$ years, which allows for comparison with previous experimental studies estimating the longitudinal change within similar transitions (Tarokh and Carskadon, 2010; Tarokh et al., 2011; Hahn et al., 2019). Sex differences in mean spindle/ $\sigma$ activity were tested using analysis of covariance at each age group. In addition, pubertal-related differences in mean spindle/ $\sigma$ activity between subjects reporting Tanner stages 1-3 $(n=56)$ versus $4-5 \quad(n=256)$ were tested at each age group, except at ages 18-22 as only one subject $\geq 18$ years reported a Tanner stage 3 and was removed from this analysis as an outlier. The results of these models are expressed as multivariable-adjusted means $(95 \% \mathrm{Cl})$. Statistical analyses were performed using SAS version 9.4 (SAS Institute).

\section{Results}

\section{Cross-sectional trajectories}

The cross-sectional sample consisted of 572 subjects aged 6-21 (truncated), of whom $48 \%$ were female and $26 \%$ were a racial/ethnic minority (Table 1 ). Mean PSG parameters were commensurate with the age range of the sample whose total sleep time (TST) was $449 \mathrm{~min}$, of which $51 \%$ was spent in N2 and $81 \%$ in NREM sleep.

As shown in Figure $1 A$, the age-related trajectory of spindle density was best fit by a quadratic model $\left(R^{2}=\right.$ 0.106 ). Spindle density was lowest at age 6.0 and highest at age 15.2; specifically, spindle density increased between ages 6 and $14\left[\beta_{\mathrm{S}}(\mathrm{SE})=0.124(0.051), p=0.014\right]$ and remained stable between ages 15 and $21\left[\beta_{\mathrm{S}}(\mathrm{SE})=\right.$ 0.009 (0.049), $p=0.852]$. The quadratic trajectory of spindle density was significant for males $\left(R^{2}=0.107\right)$, while it did not reach statistical significance in cubic, quadratic, nor linear $\left(R^{2}=0.105\right)$ models for females (Fig. $1 B$ ). Spindle density was lowest at age 6.0 and highest at age 14.2 in males. There were no statistically significant differences in spindle density between males and females when examining piece-wise age segments $6-10$ years $(p=0.100), 11-14$ years $(p=0.878), 15-17$ years $(p=0.688)$, or $18-21$ years $(p=0.223)$.

As observed in Figure $1 C$, the age-related trajectory of spindle frequency was best fit by a linear model $\left(R^{2}=\right.$ 0.212 ), by which it increased from age 6.0 (lowest) to 21.0 (highest). While this linear trajectory was significant in females $\left(R^{2}=0.246\right)$, males experienced a quadratic trajectory $\left(R^{2}=0.191\right)$ in spindle frequency (Fig. $\left.1 D\right)$. Males reached a peak in spindle frequency at age 18.0 and females at age 21.0. Commensurate, females experienced a steeper increasing slope in spindle frequency than males between ages 18 and $21\left[\beta_{\mathrm{s}}(\mathrm{SE})=0.155(0.062)\right.$ in females vs $-0.031(0.058)$ in males, $p$ for interaction $=0.013$ ] Spindle frequency was higher in females at ages 6-10 $(p=0.015)$ and at ages $18-21(p=0.006)$ but not at ages $11-$ $14(p=0.733)$ or $15-17(p=0.347)$.

The age-related trajectory for the percent of fast spindles was best fit by a quadratic model $\left(R^{2}=0.177\right.$; Fig. $1 E$ ). Fast spindle percent was lowest at age 6.0 and highest at age $19.3\left[\beta_{\mathrm{S}}\right.$ (SE) between ages 6 and $19=1.562$ $(0.450), p<0.001]$. This quadratic trajectory was significant in males $\left(R^{2}=0.173\right)$, while fast spindle percent followed a linear $\left(R^{2}=0.189\right)$ age-related trajectory in females (Fig. 1F). Although the lowest percent of fast spindles occurred at age 6.0 in males and females, males reached their peak in fast spindle percent at age 16.5 and females at age 21.0. Females showed a higher percent of fast spindles than males at ages $6-10(p=0.030)$ and 18$21(p=0.007)$ but not at ages $11-14(p=0.693)$ or $15-17$ $(p=0.809)$.

As observed in Figure 1G, the age-related trajectory of spindle power was best fit by a linear model $\left(R^{2}=0.516\right)$, as it decreased from age 6.0 (highest) to 21.0 (lowest). However, males showed a steeper decreasing slope than females from age $6-21\left[\beta_{\mathrm{S}}(\mathrm{SE})=-1.244(0.314), p\right.$ linear $<0.001 ; R^{2}=0.514$ in males vs $\beta_{\mathrm{S}}(\mathrm{SE})=-0.431$ (0.391), $p$ linear $=0.271 ; R^{2}=0.520$ in females;Fig. $\left.1 H\right]$. Spindle power was higher in females at ages $6-10$ $(p=0.045)$ and $18-21(p<0.001)$ but not at ages $11-14$ $(p=0.462)$ or $15-17(p=0.136)$.

Similar to spindle density and fast spindle percent, we found a significant quadratic trajectory $\left(R^{2}=0.235\right)$ for $\sigma$ power (Fig. 1/). However, highest $\sigma$ power was observed 
Table 1: Characteristics of the cross-sectional and longitudinal samples

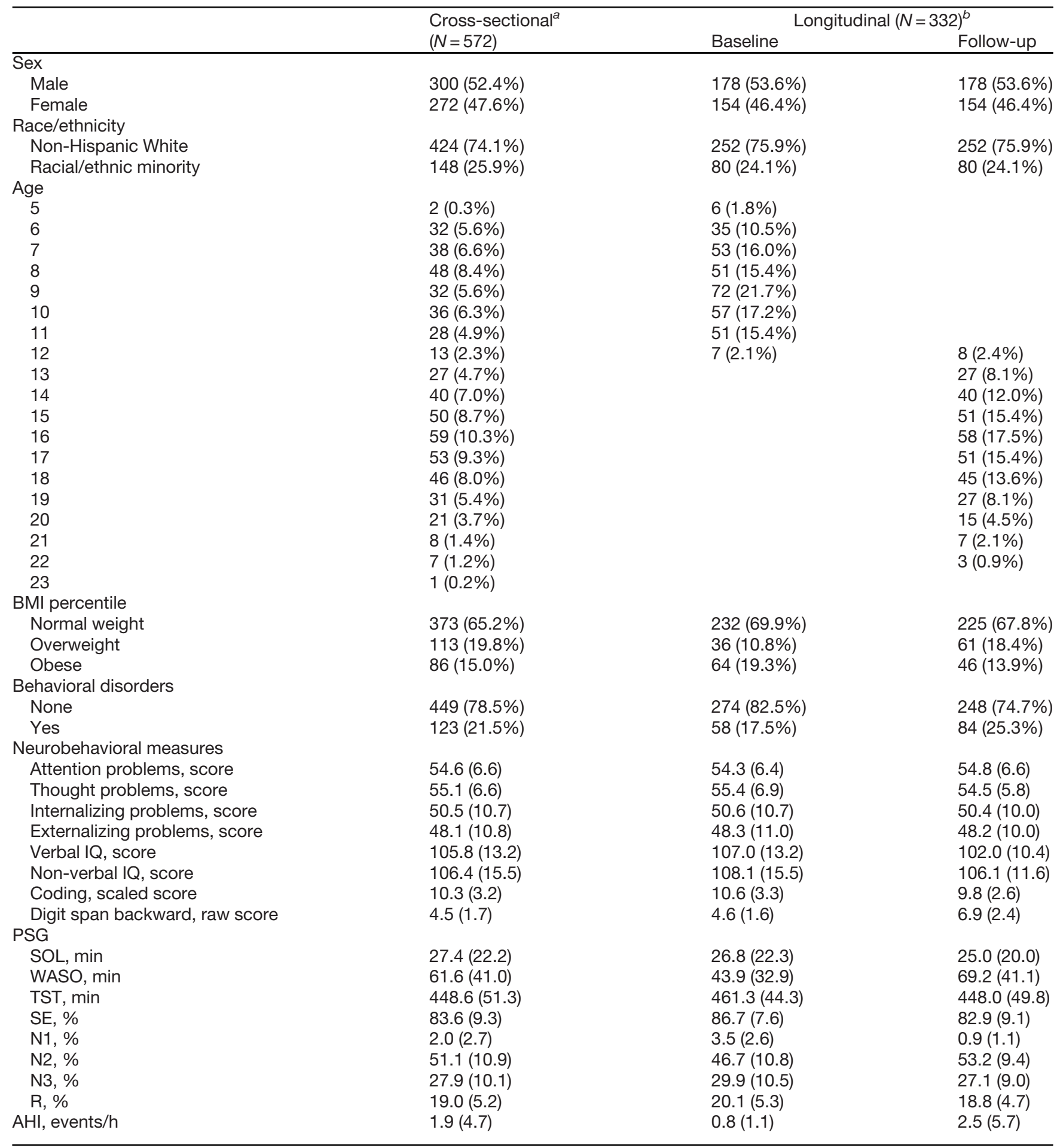

a Data for 572 subjects in cross-sectional analyses.

${ }^{\mathrm{b}}$ Data for 332 subjects in longitudinal analyses, including baseline and follow-up.

Data are means (SD) and number of cases (percentage) for continuous and categorical/ordinal variables, respectively. $\mathrm{AHI}=$ apnea/hypopnea index; BMI = body mass index; N1 = epochs scored as NREM stage 1; N2 = epochs scored as NREM stage 2; N3=epochs scored as NREM stages 3 or 4; R = epochs scored as REM sleep; SE = sleep efficiency; SOL = sleep onset latency; TST = total sleep time; WASO = wake after sleep onset.

at age 13.0 and lowest at age 21.0; specifically, $\sigma$ power decreased between ages 13 and $21\left[\beta_{\mathrm{s}}(\mathrm{SE})=73.235\right.$ (25.773), $p=0.005]$. Similar sex-related trajectories to those observed in spindle density were found for $\sigma$ power, where a quadratic trajectory $\left(R^{2}=0.226\right)$ was significant for males, while it did not reach statistical significance in cubic, quadratic nor linear $\left(R^{2}=0.246\right)$ models for females (Fig. 1J). In males, $\sigma$ power was highest at age 
A

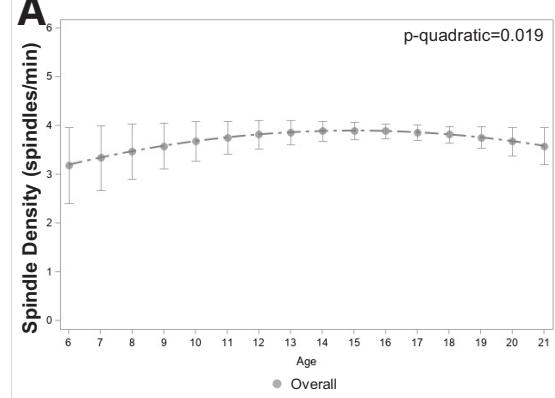

C

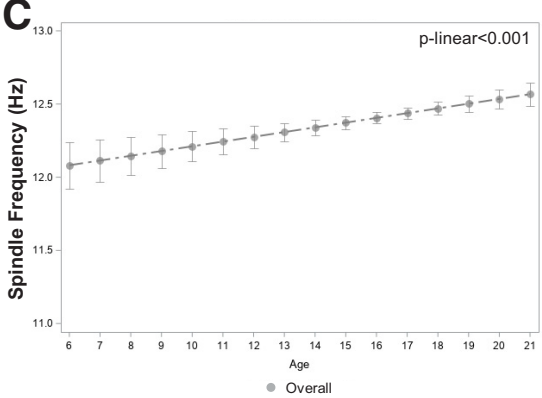

$E_{0}$

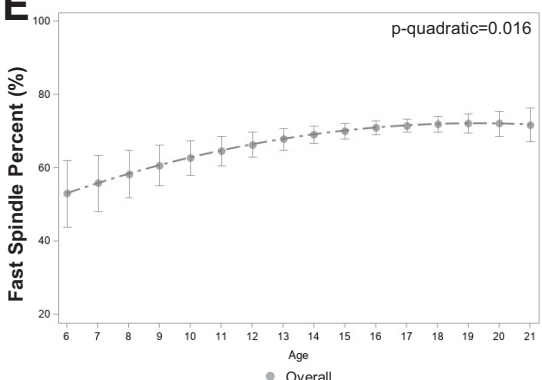

$\mathbf{G}_{\text {。 }}$

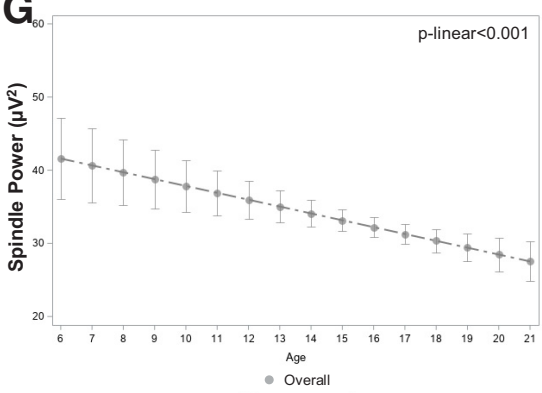

I

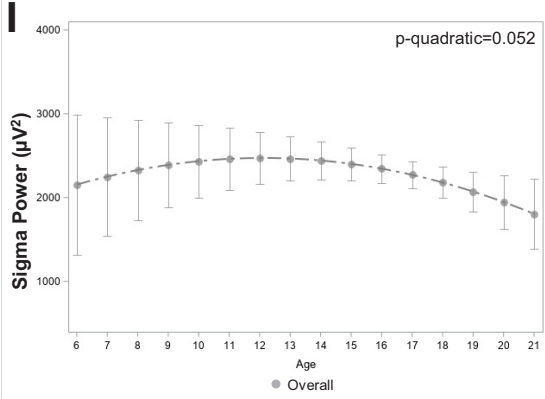

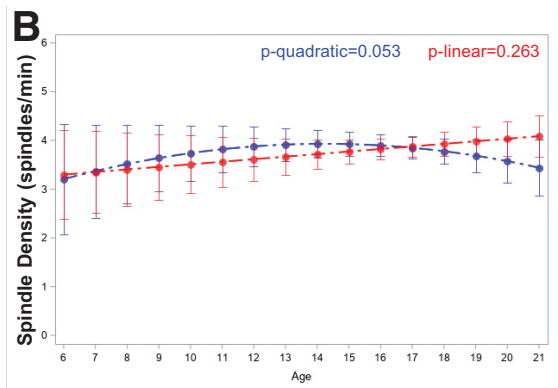

- Male $\stackrel{\text { Age }}{\text { - Female }}$
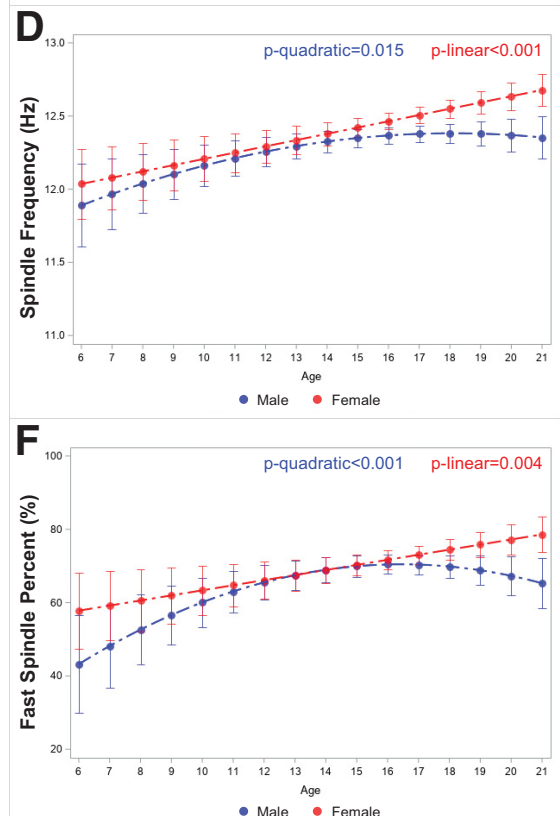

$\mathbf{H}_{s 0}$
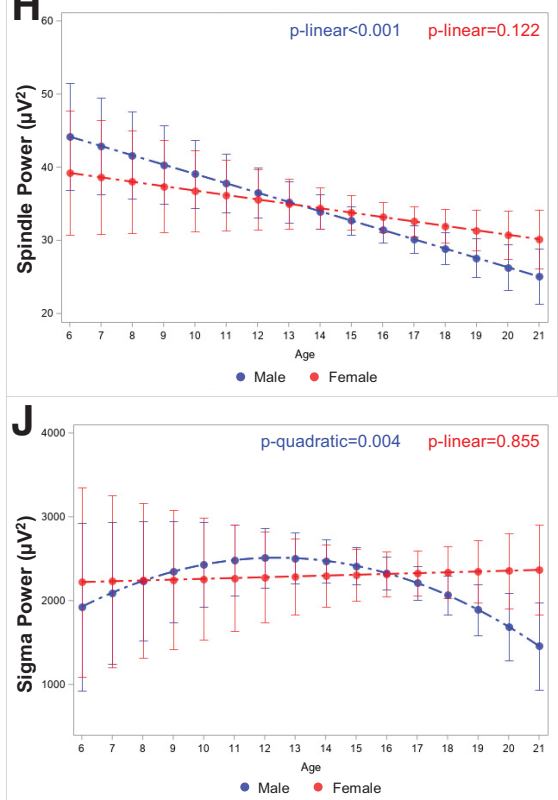

Figure 1. Cross-sectional trajectories of sleep spindles and sigma power in 572 subjects aged 6-21. Data points are multivariableadjusted means and their $95 \% \mathrm{Cl}$ and lines are multivariable-adjusted regression curves for sleep spindle and sigma activity. $\boldsymbol{A}$, Spindle density (\# of spindles/minute) overall. $\boldsymbol{B}$, Spindle density stratified by sex. $\boldsymbol{C}$, Spindle frequency $(\mathrm{Hz})$ overall. $\boldsymbol{D}$, Spindle frequency stratified by sex. $\boldsymbol{E}$, Fast spindle percent (\% of spindles in the 12-16 Hz range) overall. $\boldsymbol{F}$, Fast spindle percent stratified by sex. G, Spindle power $(\mu \mathrm{V} 2)$ overall. $\boldsymbol{H}$, Spindle power stratified by sex. I, Sigma power $(\mu \mathrm{V} 2)$ overall. J, Sigma power stratified by sex. 
Table 2: Correlation coefficients for sleep spindle/ $\sigma$ activity in the cross-sectional sample (above diagonal) and the longitudinal sample (below diagonal)

\begin{tabular}{|c|c|c|c|c|c|}
\hline & Spindle density & Spindle frequency & Fast spindle percent & Spindle power & $\sigma$ power \\
\hline Spindle density & - & 0.205 & 0.263 & 0.553 & 0.311 \\
\hline Spindle frequency & 0.286 & - & 0.931 & -0.120 & -0.104 \\
\hline Fast spindle percent & 0.349 & 0.564 & - & -0.061 & -0.106 \\
\hline Spindle power & 0.471 & 0.191 & 0.248 & - & 0.628 \\
\hline$\sigma$ power & 0.553 & 0.262 & 0.225 & 0.671 & - \\
\hline
\end{tabular}

Data are Pearson correlation coefficients. For the cross-sectional sample, the correlation coefficients for spindle density (\#/min), spindle frequency ( $\mathrm{Hz}$ ), fast spindle percent $(\%)$, and spindle and $\sigma$ power $\left(\mu \mathrm{V}^{2}\right)$ were determined. For the longitudinal sample, the correlation coefficients for the percent change in each spindle metric from baseline (ages 5-12) to follow-up (ages 12-22) were calculated.

12.6 and lowest at age 21.0. $\sigma$ power was greater in females than males at ages $6-10(p=0.033)$ and $18-21$ $(p=0.014)$ but not ages $11-14(p=0.491)$ or $15-17$ $(p=0.611)$.

Table 2 shows that, while $\sigma$ power was weakly correlated with spindle density $(r=0.311)$, it was moderately correlated with spindle power $(r=0.628)$. Spindle density and spindle power were moderately correlated to each other $(r=0.553)$, while spindle frequency was strongly correlated with fast spindle percent $(r=0.931)$.

\section{Longitudinal trajectories}

The longitudinal sample consisted of 332 children aged 5-12 at baseline (46\% female, 24\% racial/ethnic minority) who were followed-up 6-13 years later at ages 12-22 (Table 1). PSG parameters changed in the expected direction from baseline to follow-up, with TST decreasing and percent N2 increasing.

There was a significant age effect in the longitudinal change of spindle density from baseline to follow-up $\left(p<0.001 ; R^{2}=0.270\right)$. As seen in Figure $2 A$, males had experienced a greater longitudinal increase in spindle density $(33.2 \%, 95 \% \mathrm{Cl}=17.4 \%, 48.9 \%)$ than females $(2.4 \%, 95 \% \mathrm{Cl}=-14.7 \%, 19.5 \%)$ when followed-up at ages $12-14(p=0.006)$. There were no statistically significant sex differences in spindle density in the transition to ages $15-17(p=0.458)$ or $18-22(p=0.364)$.

There was an overall longitudinal increase in spindle frequency (Fig. 2B) and fast spindle percent (Fig. 2C) with no statistically significant age effect $\left(p=0.087, R^{2}=0.163\right.$ and $p=0.665, R^{2}=0.368$, respectively). Females had experienced a greater longitudinal increase in spindle frequency $(3.4 \%, 95 \% \mathrm{Cl}=2.5 \%, 4.3 \%)$ than males $(1.7 \%$, $95 \% \mathrm{Cl}=0.8 \%, 2.7 \%)$ when followed-up at ages $18-22$ $(p=0.004$; Fig. 2B). Further, females had experienced a greater increase in fast spindle percent $(66.4 \%, 95 \%$ $\mathrm{Cl}=45.4 \%, 87.4 \%)$ than males $(25.2 \%, 95 \% \mathrm{Cl}=2.6 \%$, $47.9 \%)$ when followed-up at ages 18-22 ( $p=0.004$; Fig. $2 C)$. There were no statistically significant sex differences in spindle frequency nor fast spindle percent in the transitions to ages $12-14(p=0.598$ and $p=0.759$, respectively) nor $15-17$ ( $p=0.795$ and $p=0.833$, respectively).

There was an overall longitudinal decline in spindle power with a significant age effect $\left(p<0.001 ; R^{2}=\right.$ 0.330). Males had experienced a greater decline in spindle power $(-53.4 \%, 95 \% \mathrm{Cl}=-62.7 \%,-44.0 \%)$ than females $(-39.1 \%, 95 \% \mathrm{Cl}=-47.7 \%,-30.5 \%)$ when followed-up at ages 18-22 ( $p=0.018$; Fig. 2D).
There were no statistically significant sex differences in spindle power in the transitions to ages 12-14 $(p=0.265)$ nor $15-17(p=0.341)$.

There was also a significant age effect $(p<0.001$; $R^{2}=0.264$ ) in the longitudinal change in $\sigma$ power from baseline to follow-up. As shown in Figure $2 E$, males had experienced a $26 \%$ greater increase in $\sigma$ power $(18.3 \%, 95 \% \mathrm{Cl}=1.4 \%, 35.2 \%)$ than females $(-7.4 \%$, $95 \% \mathrm{Cl}=-24.7 \%, 10.0 \%)$ when followed-up at ages 12-14 $(p=0.032)$. There were no statistically significant sex differences in $\sigma$ power in the transitions to ages 15-17 $(p=0.291)$ nor 18-22 $(p=0.280)$.

We also examined the longitudinal change in spindle and $\sigma$ activity as a function of Tanner stage at follow-up. As observed in Figure $2 F$, subjects who reported Tanner stages 1-3 had experienced a greater longitudinal increase in spindle density $(37.9 \%, 95 \% \mathrm{Cl}=20.2 \%, 55.6 \%)$ than those reporting Tanner stages $4-5(1.9 \%, 95 \% \mathrm{Cl}=-13.5 \%, 17.4 \%)$ when followed-up at ages 12-14 $(p=0.002)$ but not at ages 15-17 $(p=0.575)$. As seen in Figure $2 G, H$, there were no significant differences between subjects reporting Tanner stages 1-3 versus $4-5$ at ages $12-14$ or 15-17 in the longitudinal change of spindle frequency ( $p=0.576$ and $p=0.340$, respectively) or fast spindle percent $(p=0.661$ and $p=0.432$, respectively). Subjects who reported Tanner stages 4-5 had experienced a $14 \%$ greater longitudinal decline in spindle power $(-35.7 \%$, $95 \% \mathrm{Cl}=-45.2 \%,-26.1 \%)$ than those reporting Tanner stages $1-3(-21.9 \%, 95 \% \mathrm{Cl}=-32.5 \%,-11.3 \%)$ when followed-up at ages $12-14(p=0.055)$ but not at ages 15-17 ( $p=0.769$; Fig. 2l). Lastly, subjects who reported Tanner stages 4-5 had experienced a 34\% greater longitudinal decline in $\sigma$ power $(-28.8 \%, 95 \% \mathrm{Cl}=-53.8 \%$, $-3.7 \%)$ than those reporting Tanner stages $1-3(5.0 \%$, $95 \% \mathrm{Cl}=-23.0 \%, 33.0 \%)$ when followed-up at ages 12$14(p=0.008)$ but not at ages 15-17 ( $p=0.448$; Fig. 2J).

Finally, as shown in Table 2, the longitudinal change in $\sigma$ power was moderately correlated with both the change in spindle density $(r=0.553)$ and in spindle power $(r=0.671)$ from baseline to follow-up. The change in spindle density was weakly correlated with the change in spindle power $(r=0.471)$, while the changes in spindle frequency and fast spindle percent were moderately correlated $(r=0.564)$ to each other.

\section{Discussion}

This study delineates sex-related and pubertal-related differences in the maturational trajectories of sleep spindles in a population-based cohort capturing the critical 

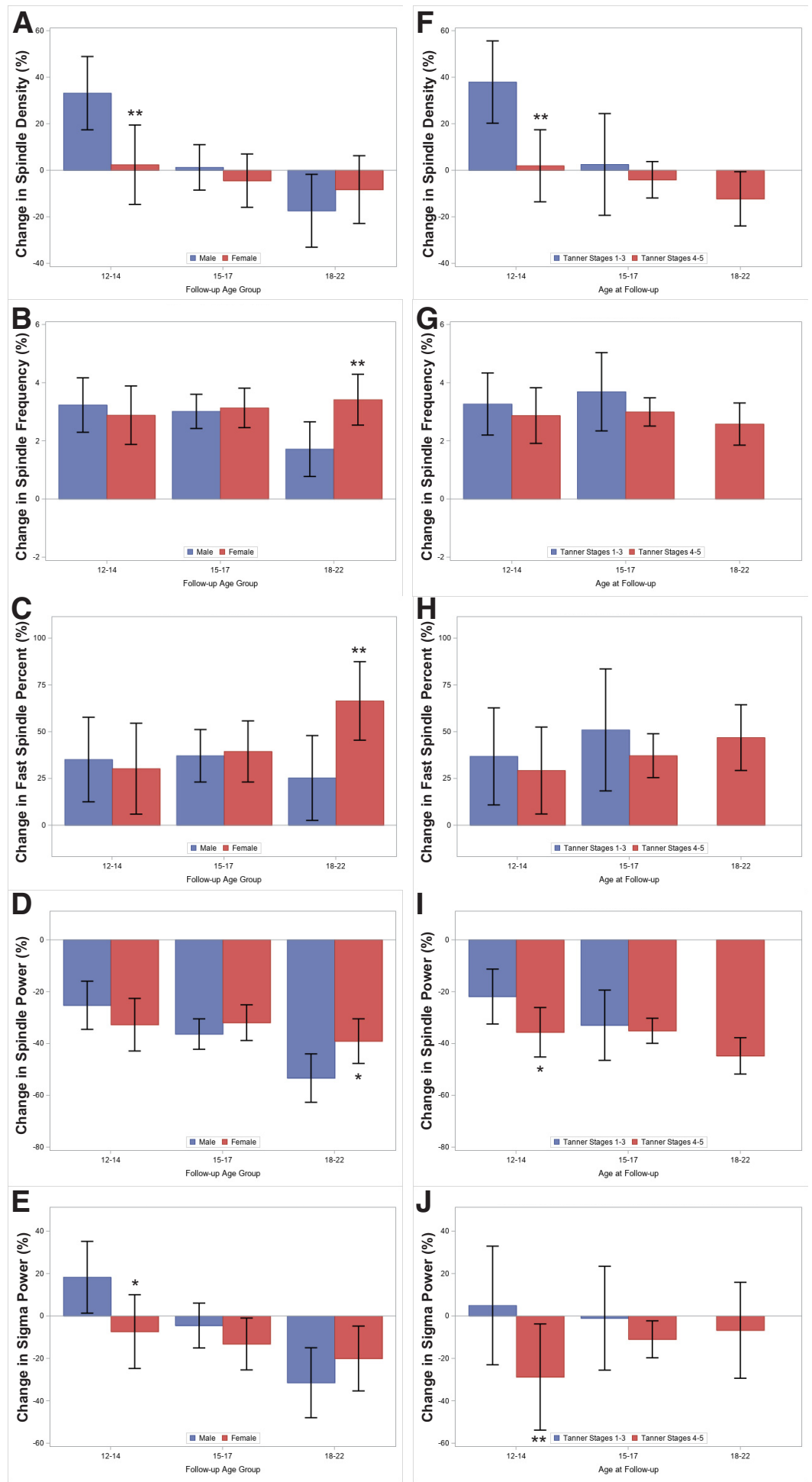

Figure 2. Sex (left column) and Tanner-related (right column) differences in the longitudinal trajectories of sleep spindles and sigma power in 332 subjects aged 5-12 at baseline followed-up at ages 12-22. Data are multivariable-adjusted percent change (95\% Cl) from baseline to follow-up in sleep spindle and sigma activity. Age groups represent transitions between baseline age (mean) 6.6 years to follow-up ages $12-14$, from 8.7 years to ages $15-17$, and from 10.3 years to ages $18-22$. $\boldsymbol{A}$, Spindle density stratified by sex. $\boldsymbol{B}$, Spindle frequency stratified by sex. $\boldsymbol{C}$, Fast spindle percent stratified by sex. $\boldsymbol{D}$, Spindle power stratified by sex. $\boldsymbol{E}, \sigma$ power stratified by sex. $\boldsymbol{F}$, Spindle density stratified by Tanner stage. $\boldsymbol{G}$, Spindle frequency stratified by Tanner stage. $\boldsymbol{H}$, Fast spindle percent stratified by Tanner stage. I, Spindle power stratified by Tanner stage. $\boldsymbol{J}, \sigma$ power stratified by Tanner stage. ${ }^{*} p<0.05$, ${ }^{* *} p<0.01$. 
developmental period between childhood and adolescence. We provide robust evidence that sleep spindle metrics follow distinct developmental trajectories that deviate from other sleep EEG oscillations previously associated with brain maturation, namely slow wave activity (SWA) in the $\delta$-frequency $(0.4-4.0 \mathrm{~Hz})$ range (Baker et al., 2012; Campbell et al., 2012; Campbell and Feinberg, 2016; Gorgoni et al., 2020; Ricci et al., 2021). Specifically, spindle density, fast spindle percent, and $\sigma$ power, follow inverted U-shaped trajectories from age 6 to 21 , by which both spindle density and $\sigma$ power increase during childhood, peak in mid-adolescence, and begin to decline in late adolescence/early adulthood. While the percent of fast spindles also increases in childhood, it continues to increase further into late adolescence, commensurate with the linear increase in spindle frequency from age 6 to 21 . In contrast, spindle power linearly declines from childhood to early adulthood. Overall, our novel data show that males experience greater maturational changes in spindle density, spindle power and $\sigma$ power, which is driven by their later pubertal development, yet sex differences become prominent in early adulthood when males have lower spindle frequency, spindle power and $\sigma$ power as well as a lower percentage of fast spindles.

Our observed inverted U-shaped cross-sectional trajectory of spindle density from childhood to early adulthood replicates previous life-course analyses by Purcell et al. (2017), by which spindle density increases in childhood, peaks in adolescence, and starts to decline in adulthood. Although both $\sigma$ power and spindle density increase during childhood and peak in adolescence, the peak in $\sigma$ power occurs two years earlier (age 13) than spindle density (age 15). In addition, $\sigma$ power declines to a greater extent in early adulthood and does not reach its lowest until age 21, while lowest spindle density occurs at age 6. Further, the decline in $\sigma$ power in late adolescence/ early adulthood is consistent with and may be related to the decline in spindle power observed through age 21 as they are moderately correlated. Thus, although $\sigma$ power may undergo similar age-related changes to spindle density and spindle power, using $\sigma$ power as a surrogate marker for all spindle metrics may mask specific age-related changes in important spindle metrics, including density, as shown by the small to moderate correlations in Table 2 between $\sigma$ power and spindle activity (Goldstone et al., 2019; Gorgoni et al., 2020).

From a neurobiological standpoint, the observed increase in spindle density up to mid-adolescence may represent the increased myelination of thalamocortical projections that occurs with typical neurodevelopment (Steriade et al., 1985; Tarokh et al., 2011), as neuroimaging studies have shown higher spindle density to be associated with enhanced white matter diffusion along axons (Piantoni et al., 2013). Additionally, Hahn et al. (2019) suggested that the increase in spindle density in adolescence may be related to the emergence of centroparietal fast spindles. Consistently, the inverted-U shaped trajectory in spindle density coupled with the linear increase in spindle frequency was reflected in the percent of fast spindles also increasing in early and mid-adolescence and peaking by age 19, suggesting that "full" maturation of the thalamocortical network may occur by late adolescence, consistent with previous neuroimaging studies examining maturational changes in cortical gray and white matter (Giedd et al., 1999; Giorgio et al., 2010). Furthermore, the increase in spindle frequency from childhood to early adulthood is hypothesized to reflect reduced hyperpolarization of thalamocortical neurons that occurs with the decline in sleep depth throughout adolescence (Campbell and Feinberg, 2016; Goldstone et al., 2019). This reduced hyperpolarization of thalamocortical projections increases the number of neurons able to produce spindles, thus, allowing for greater spindle density and spindle frequency (Steriade et al., 1985; Goldstone et al., 2019). Further, Saletin et al. (2013) found fast $(13-15 \mathrm{~Hz})$ spindle frequencies to be positively associated with hippocampal gray matter volume, which was a predictor of increasingly fast frequency spindles in 22 young adults. Fast spindles have also been associated with hippocampal-dependent memory consolidation (Diekelmann and Born, 2010), thus, the increase in spindle frequency and percent of fast spindles throughout childhood and adolescence may also reflect maturation of hippocampal connectivity (Saletin et al., 2013; Astill et al., 2014). Our observed age-related trajectories of spindle density, frequency, and percent of fast spindles, taken together with previous EEG and neuroimaging studies, further support the role of sleep spindles as EEG biomarkers of thalamocortical and hippocampal development.

An important novel aspect of our study was examining within-subjects changes in spindle metrics as a function of pubertal development, as previous studies have been limited by short follow-up periods in childhood and adolescence and a lack of pubertal data (Purcell et al., 2017; Hahn et al., 2019). Adolescents reporting more mature pubertal stages experienced a greater decline in $\sigma$ and spindle power than less mature adolescents in the transition from childhood (ages 5-8, mean 6.6 years) to early adolescence (ages 12-14, mean 13.4 years). These data suggest that changes in $\sigma$ and spindle power may be closely related to pubertal development similar to SWA power, a well-described marker of brain maturation, specifically synaptic pruning (Baker et al., 2012; Feinberg and Campbell, 2012; Ricci et al., 2021). This pubertal-related decline in $\sigma$ and spindle power observed in more mature youth in the transition to early adolescence suggests a potential role for synaptic pruning, which may be impacting the synchronization of slow wave and sleep spindle oscillations at the cortical level because of their similar origin in the thalamocortical network (Steriade, 2006; Campbell and Feinberg, 2016; Gorgoni et al., 2020). Furthermore, spindle and $\sigma$ power have been associated with subcortical white matter integrity around the thalamus in adults (Piantoni et al., 2013; Gaudreault et al., 2018; Fernandez and Lüthi, 2020); thus, an additional neurobiological mechanism that may be driving the decline in spindle and $\sigma$ power in adolescence may be reduced diffusivity around the neuronal membrane, which leads to greater neural synchrony in thalamocortical loops (Gaudreault et al., 2018). Although both spindle and $\sigma$ 
power decline in the transition to adolescence, spindle power continues to decline further into late adolescence/ early adulthood in mature subjects $(-45 \%$ decline in spindle power by 18-22 years vs $-7 \%$ in $\sigma$ power). Taken together, these data suggest that the decrease in $\sigma$ power may be more related to cortical synaptic pruning in the transition to adolescence, while the decline in spindle power may reflect the continued maturation of subcortical white matter (Campbell and Feinberg, 2016; Gaudreault et al., 2018). We also found that adolescents reporting less mature pubertal stages, $86 \%$ of whom were males, had experienced a greater increase in spindle density in the transition to early adolescence (age 12-14), while more mature adolescents, $75 \%$ of whom were females, had already experienced such increase in spindle density. Overall, these data suggest that females, who enter pubertal development earlier than males, may also begin brain maturational processes in the thalamocortical network at an earlier stage than males (Giedd et al., 1999; Colrain and Baker, 2011). Interestingly, there were no significant pubertal-related differences in spindle frequency nor percent of fast spindles, suggesting that while the quantity (density) of spindles generated by the thalamus is associated with pubertal development, the quality (frequency) of spindles expressed at the cortical level may not (Anderer et al., 2001; Saletin et al., 2013). Future studies examining age-related changes in cortical and subcortical gray and white matter and sleep spindle/ $\sigma$ activity are needed to shed further light on the association of spindle/ $\sigma$ activity with specific brain maturational processes.

As it pertains to sex differences, males appeared to drive the inverted $U$-shaped cross-sectional trajectories of spindle density, fast spindle percent, and $\sigma$ power. Females maintained more stable levels of spindle density and $\sigma$ power from childhood into early adulthood, while males experienced greater maturational changes. Although males reached peak $\sigma$ power and spindle density at ages 12.6 and 14.2, they experienced steeper declines throughout late adolescence/early adulthood leading to lower $\sigma$ power than females, consistent with Purcell et al. (2017). The longitudinal change in spindle density by ages 12-14 was lower in females than males, suggesting that females had already experienced their maximum spindle density before the transition to early adolescence, commensurate with our observed pubertal differences. Similarly, males appeared to drive the linear decline in spindle power in late adolescence/early adulthood, as they exhibited a steeper maturational slope and greater longitudinal decline than females by ages 1822. Together, these findings indicate that the age-related trajectories of spindle density, spindle power and $\sigma$ power are associated with the onset of puberty, which occurs earlier in females than males (Giedd et al., 1999; Colrain and Baker, 2011). Thus, females may mature their spindle characteristics at earlier developmental stages, while males experience greater maturational changes through early adulthood, consistent with a previous study by Ujma et al. (2016), suggesting that spindle density is a maturational marker in males, but may be a trait-like EEG feature in females. Interestingly, females experienced a linear increase in spindle frequency, leading them to have a higher percentage of fast spindles than males by early adulthood, commensurate with previous studies in adolescents and adults (Purcell et al., 2017; Goldstone et al., 2019). While the percent of fast spindles plateaued in males by age 17, it continued to increase in females through age 21. These data suggest that females may have greater thalamocortical connectivity and coherence than males by early adulthood (Markovic et al., 2020). Overall, the observed sex differences in spindle activity indicate that there may be potential differences in thalamocortical loops in females and males (Markovic et al., 2020); however, future studies that examine brain connectivity, cognitive processes, and sleep spindles are needed to shed further light on the functional significance of the observed maturational sex differences.

Several potential limitations of the present study must be discussed. Sleep studies consisted of a one-night PSG recording, which may be affected by the first night effect; however, spectral EEG measures have shown greater night-to-night stability than traditional sleep continuity/architecture parameters, assuring the generalizability of the findings (Tan et al., 2000; Israel et al., 2012). Although we were able to control for AHI, immediate sleep history during the week previous to the in-lab study was not available and standardized across all age groups, and thus, could not be controlled for in the analyses. Additionally, there were PSG system updates during this long-term study; however, we accounted for each setting during EEG data processing and controlled for the PSG system used in statistical analyses to mitigate the potential impact on the estimation and trajectories of sleep spindles and $\sigma$ activity. The lack of a significant age term (linear, quadratic, or cubic) for spindle density, spindle power or $\sigma$ power in females, may be because of a lack of statistical power. Although some subjects contributed with ipsilateral central derivations at ages 5-12 and and the distance between active and reference electrode can affect the amplitude of spectral bands, there was excellent concordance between contralateral and ipsilateral derivations, assuring the validity of our data (see Materials and Methods, PSG). Our longitudinal analyses in 332 subjects were based on two time-points, which precluded analyzing the data with longitudinal growth curves. Although we had Tanner staging available at follow-up, it was not collected at baseline; however, the age-sex-Tanner distribution was that expected for a population-based sample where females mature earlier than males (Campbell et al., 2012), assuring the generalizability of our findings. Additionally, we focused our analyses on central derivations as frontal derivations (e.g., F3-M2) were not available at both time points, which precluded topographical analysis of spindle metrics in the cross-sectional and longitudinal trajectories for either the entirety of the 6-21 life course or in the transition from ages 5-12 to ages 12-22. However, spindle activity has been shown to be best quantified at central derivations (Goldschmied et al., 2020), assuring the reliability of our findings. Finally, although we were able to calculate the percent of fast spindles, MSS 
software did not allow estimating the density, power, or peak frequency of fast and slow spindles separately, thus, future studies are necessary to determine the agerelated trajectories of those specific characteristics.

In conclusion, we provide population-based evidence that sleep spindle metrics follow distinct developmental trajectories from each other and from other synchronized oscillations of NREM sleep previously shown to index brain maturation (e.g., SWA power) during the critical transition from childhood to adolescence. Although sleep spindles occur within the $\sigma$-frequency range, it appears that using $\sigma$ power as a surrogate marker for spindle activity may mask spindle-specific maturational changes. The increase in spindle density in childhood and peak in mid-adolescence coupled with the linear increase in spindle frequency may represent the increasing predominance of fast spindles and, thus, maturation of thalamocortical and hippocampal connectivity, which appears to continue until late adolescence. The greater longitudinal decline in $\sigma$ and spindle power in more mature adolescents as well as the greater increase in spindle density in less mature subjects by early adolescence indicates that females, who enter pubertal development earlier than males, may begin maturation of their thalamocortical loops earlier than males. Indeed, females had higher spindle frequency, spindle power and $\sigma$ power and a greater percentage of fast spindles by early adulthood, which may reflect greater thalamocortical coherence, while males experience greater maturational changes in spindle density, spindle power and $\sigma$ power throughout childhood and adolescence. These data have important implications for future studies examining the role of sleep spindle activity in specific psychiatric and/or learning disorders as well as their relationship to cognitive abilities in youth at different developmental stages.

\section{References}

Achenbach TM (1991) Integrative guide for the 1991 CBCL/4-8, YSR, and TRF profiles. Burlington: Research Center for Children, Youth, and Families, Department of Psychiatry, University of Vermont.

Achenbach TM, Rescorla LA (2003) Manual for the ASEBA adult forms and profiles. Burlington: Research Center for Children, Youth, and Families, Department of Psychiatry, University of Vermont.

Anderer P, Klösch G, Gruber G, Trenker E, Pascual-Marqui RD, Zeitlhofer J, Barbanoj MJ, Rappelsberger P, Saletu B (2001) Lowresolution brain electromagnetic tomography revealed simultaneously active frontal and parietal sleep spindle sources in the human cortex. Neuroscience 103:581-592.

Astill RG, Piantoni G, Raymann RJEM, Vis JC, Coppens JE, Walker MP, Stickgold R, Van Der Werf YD, Van Someren EJW (2014) Sleep spindle and slow wave frequency reflect motor performance in primary school-age children. Front Hum Neurosci 8:e00910.

Baker F, Turlington SR, Colrain IM (2012) Developmental changes in the sleep electroencephalogram of adolescent boys and girls. J Sleep Res 21:59-67.

Baker FC, Willoughby AR, de Zambotti M, Franzen PL, Prouty D, Javitz H, Hasler B, Clark DB, Colrain IM (2016) Age-related differences in sleep architecture and electroencephalogram in adolescents in the national consortium on alcohol and neurodevelopment in adolescence sample. Sleep 39:1429-1439.

Bixler EO, Vgontzas AN, Lin HM, Liao D, Calhoun SL, Fedok F, Vlasic V, Graff G (2008) Blood pressure associated with sleep-disordered breathing in a population sample of children. Hypertension 52:841-846.

Bixler EO, Fernandez-Mendoza J, Liao D, Calhoun SL, RodriguezColon SM, Gaines J, He F, Vgontzas AN (2016) Natural history of sleep disordered breathing in prepubertal children transitioning to adolescence. Eur Respir J 67:1402-1409.

Bòdizs R, Gombos F, Ujma PP, Kovács I (2014) Sleep spindling and fluid intelligence across adolescent development: sex matters. Front Hum Neurosci 28:952.

Calhoun SL, Fernandez-Mendoza J, Vgontzas AN, Liao D, Bixler EO (2014) Prevalence of insomnia symptoms in a general population sample of young children and preadolescents: gender effects. Sleep Med 15:91-95.

Campbell IG, Feinberg I (2016) Maturational patterns of $\sigma$ frequency power across childhood and adolescence: a longitudinal study. Sleep 39:193-201.

Campbell IG, Darchia N, Khaw WY, Higgins LM, Feinberg I (2005) Sleep EEG evidence of sex differences in adolescent brain maturation. Sleep 28:637-643.

Campbell IG, Grimm KJ, de Bie E, Feinberg I (2012) Sex, puberty, and the timing of sleep EEG measured adolescent brain maturation. Proc Natl Acad Sci USA 109:5740-5743.

Carskadon MA, Acebo C (1993) A self-administered rating scale for pubertal development. J Adolesc Health 14:190-195.

Chatburn A, Coussens S, Lushington K, Kennedy D, Baumert M, Kohler M (2013) Sleep spindle activity and cognitive performance in healthy children. Sleep 36:237-243.

Colrain IM, Baker F (2011) Changes in sleep as a function of adolescent development. Neuropsychol Rev 21:5-21.

Dang-Vu TT, Mckinney SM, Buxton OM, Solet JM, Ellenbogen JM (2010) Spontaneous brain rhythms predict sleep stability in the face of noise. Curr Biol 20:R626-R627.

Dang-Vu TT, Salimi A, Boucetta S, Wenzel K, O’Byrne J, Brandewinder M, Berthomier C, Gouin JP (2015) Sleep spindles predict stress-related increases in sleep disturbances. Front Hum Neurosci 9:68.

D’Atri A, Novelli L, Ferrara M, Bruni O, De Gennaro L (2018) Different maturational changes of fast and slow sleep spindles in the first four years of life. Sleep Med 42:73-82.

Diekelmann S, Born J (2010) The memory function of sleep. Nat Rev Neurosci 11:114-126.

Feinberg I, Campbell IG (2012) Longitudinal sleep EEG trajectories indicated complex patterns of adolescent brain maturation. Am J Physiol Regul Integr Comp Physiol 304:R296-R303.

Fernandez LMJ, Lüthi A (2020) Sleep spindles: mechanism and functions. Physiol Rev 100:805-868.

Fernandez-Mendoza J, Li Y, Vgontzas AN, Fang J, Gaines J, Calhoun SL, Liao D, Bixler EO (2016) Insomnia is associated with cortical hyperarousal as early as adolescence. Sleep 39:10291036.

Fernandez-Mendoza J, Baker JH, Vgontzas AN, Gaines J, Liao D, Bixler EO (2017) Insomnia symptoms with objective short sleep duration are associated with systemic inflammation in adolescents. Brain Behav Immun 61:110-116.

Fernandez-Mendoza J, Li Y, Fang J, Calhoun SL, Vgontzas AN, Liao D, Bixler EO (2019) Childhood high-frequency EEG activity during sleep is associated with incident insomnia symptoms in adolescence. J Child Psychol Psychiatry 60:742-751.

Fogel SM, Smith CT (2011) The function of the sleep spindle: a physiological index of intelligence and a mechanism for sleep-dependent memory consolidation. Neurosci Biobehav Rev 35:1154-1165.

Frye SS, Fernandez-Mendoza J, Calhoun SL, Vgontzas AN, Liao D, Bixler EO (2018) Neurocognitive and behavioral significance of periodic limb movements during sleep in adolescents with attention-deficit/hyperactivity disorder. Sleep 41:zsy129.

Gaudreau H, Carrier J, Montplaisir J (2001) Age-related modifications of NREM sleep EEG: from childhood to middle age. J Sleep Res 10:165-172.

Gaudreault P, Gosselin N, Lafortune M, Deslauriers-Gauthier S, Martin N, Bouchard M, Dube J, Lina J, Doyon J, Carrier J (2018) 
The association between white matter and sleep spindles differs in young and older individuals. Sleep 41:zsy113.

Geiger A, Huber R, Kurth S, Ringli M, Jenni OG, Achermann P (2011) The sleep EEG as a marker of intellectual ability in school age children. Sleep 34:181-189.

Giedd JN, Blumenthal J, Jeffries NO, Castellanos FX, Liu H, Zijdenbos A, Paus T, Evans AC, Rapoport JL (1999) Brain development during childhood and adolescence: a longitudinal MRI study. Nat Neurosci 2:861-863.

Giorgio A, Watkins KE, Chadwick M, James S, Winmill L, Douaud G, De Stefano N, Matthews PM, Smith SM, Johansen-Berg H, James AC (2010) Longitudinal changes in grey and white matter during adolescence. Neuroimage 49:94-103.

Goldschmied JR, Lacourse K, Maislin G, Delfrate J, Gehrman P, Pack FM, Staley B, Pack Al, Younes M, Kuna ST, Warby SC (2020) Spindles are highly heritable as identified by different spindle detectors. Sleep 44:zsaa230.

Goldstone A, Willoughby AR, de Zambotti M, Clark DB, Sullivan EV, Hasler BP, Franzen PL, Prouty DE, Colrain IM, Baker FC (2019) Sleep spindle characteristics in adolescents. Clin Neurophysiol 130:893902.

Gorgoni M, D'Atri A, Scarpelli S, Reda F, De Gennaro L (2020) Sleep electroencephalography and brain maturation: developmental trajectories and the relation with cognitive functioning. Sleep Med 66:33-50.

Gruber R, Wise MS (2016) Sleep spindle characteristics in children with neurodevelopmental disorders and their relation to cognition. Neural Plast 2016:4724792.

Guadagni V, Byles H, Hanly PJ, Younes M, Poulin M (2019) Spindle characteristics are associated with executive function in healthy older adults from the brain in motion study. Sleep 42:A37-A38.

Guadagni V, Byles $H$, Tyndall AV, Parboosingh J, Longman RS, Hogan DB, Hanly P, Younes M, Poulin MJ (2021) Association of sleep spindle characteristics with executive functioning in healthy sedentary middle-aged and older adults. J Sleep Res 30:e13037.

Hahn M, Joechner AK, Roell J, Schabus M, Heib DPJ, Gruber G, Peigneux P, Hoedlmoser K (2019) Developmental changes of sleep spindles and their impact on sleep-dependent memory consolidation and general cognitive abilities: a longitudinal approach. Dev Sci 22:e12706.

Hoedlmoser K, Heib DPJ, Roell J, Peigneux P, Sadeh A, Gruber G, Schabus M (2014) Slow sleep spindle activity, declarative memory, and general cognitive abilities in children. Sleep 37:1501-1512.

Iber C, Ancoli-Israel S, Chesson AL, Quan SF (2007) The AASM manual for the scoring of sleep and associated events: rules, terminology and technical specifications. Westchester: The American Academy of Sleep Medicine.

Israel B, Buysse DJ, Krafty RT, Begley A, Miewald J, Hall M (2012) Short-term stability of sleep and heart rate variability in good sleepers and patients with insomnia: for some measures, one night is enough. Sleep 35:1285-1291.

Kuczmarski RJ, Ogden CL, Guo SL, Grummer-Strawn LM, Flegal KM, Mei Z, Wei R, Curtin LR, Roche AF, Johnson CL (2002) 2000 CDC growth charts for the United States: methods and development. Vital Health Stat 246:1-190.

Markovic A, Kaess M, Tarokh L (2020) Gender differences in adolescent sleep neurophysiology: a high density sleep EEG study. Sci Rep 10:15935.

McClain IJ, Lustenberger C, Achermann P, Lassonde JM, Kurth S, LeBourgeois MK (2016) Developmental changes in sleep spindle characteristics and $\sigma$ power across early childhood. Neural Plast 2016:3670951.

Nader RS, Smith CT (2015) Correlations between adolescent processing speed and specific spindle frequencies. Front Hum Neurosci 9:30.

Olbrich E, Rusterholz T, LeBourgeois MK, Achermann P (2017) Developmental changes in sleep oscillations during early childhood. Neural Plast 2017:6160959.

Piantoni G, Poil SS, Linkenkaer-Hansen K, Verweij LM, Ramautar JR, Van Someren EJ, Van Der Werf YD (2013) Individual differences in white matter diffusion affect sleep oscillations. J Neurosci 33:227233.

Prehn-Kristensen A, Munz M, Molzow I, Wilhelm I, Wiesner CD, Baving $L$ (2013) Sleep promotes consolidation of emotional memory in healthy children but not in children with attention-deficit hyperactivity disorder. PLoS One 8:e65098.

Purcell SM, Manoach DS, Demanuele C, Cade BE, Mariani S, Cox R, Panagiotaropoulou G, Saxena R, Pan JQ, Smoller JW, Redline S, Stickgold R (2017) Characterizing sleep spindles in 11,630 individuals from the national sleep research resource. Nat Commun 8:15930.

Rechtschaffen A, Kales A (1968) A manual of standardized terminology, techniques and scoring system for sleep stages of human subjects, p 204. Bethesda: National Institutes of Health.

Reynolds CM, Short MA, Gradisar M (2018) Sleep spindles and cognitive performance across adolescence: a meta-analytic review. J Adolesc 66:55-70.

Ricci A, He F, Fang J, Calhoun SL, Vgontzas AN, Liao D, Younes M, Bixler EO, Fernandez-Mendoza J (2021) Maturational trajectories of non-rapid eye movement slow wave activity and odds ratio product in a population-based sample of youth. Sleep Med 83:271-279.

Saletin JM, van der Helm E, Walker MP (2013) Structural brain correlates of human sleep oscillations. Neuroimage 83:658-668.

Steriade M (1999) Brainstem activation of thalamocortical systems. Brain Res Bull 50:391-392.

Steriade M (2006) Grouping of brain rhythms in corticothalamic systems. Neuroscience 137:1087-1106.

Steriade M, Deschênes M, Domich L, Mulle C (1985) Abolition of spindle oscillations in thalamic neurons disconnected from nucleus reticularis thalami. J Neurophysiol 54:1473-1497.

Tan X, Campbell IG, Palagini L, Feinberg I (2000) High internight reliability of computer-measured NREM delta, $\sigma$, and beta: biological implications. Biol Psychiatry 48:1010-1019.

Tarokh L, Carskadon MA (2010) Developmental changes in the human sleep EEG during early adolescence. Sleep 33:801-809.

Tarokh L, Carskadon MA, Achermann P (2011) Trait-like characteristics of the sleep EEG across adolescent development. J Neurosci 31:6371-6378.

Ujma PP, Sándor P, Szakadát S, Gombos F, Bódizs R (2016) Sleep spindles and intelligence in early childhood-developmental and trait-dependent aspects. Dev Psychol 52:2118-2129.

Wechsler D (1991) Wechsler intelligence scale for children (WISC-III), Ed 3. San Antonio: The Psychological Corporation.

Wechsler D (1997) Wechsler adult intelligence scale (WAIS-III), Ed 3. San Antonio: The Psychological Corporation.

Wechsler D (1999) Wechsler abbreviated scale of intelligence (WASI). San Antonio: The Psychological Corporation.

Wechsler D (2003) Wechsler intelligence scale for children (WISC-IV), Ed 4. San Antonio: The Psychological Corporation. 